Article

\title{
A method to compute the determinant of square matrices of order five and six
}

\section{Armend Salihu1,*}

1 South East European University, Ilindenska no. 335, 1200 Tetovo, Macedonia.

* Correspondence: ar.salihu@gmail.com

Received: 13 September 2018; Accepted: 11 March 2019; Published: 1 April 2019.

Abstract: In this paper we present a new method to compute the determinants of square matrices of order 5 and 6. To prove the main results we have combined the Farhadian's Duplex Fraction method and Salihu's method to reduce the order of determinants to second order. Hence, this paper gives the possibility to develop a general method to compute the determinants of higher order.

Keywords: Determinants, Farhadian's Duplex fraction, twice Dodgson's condensation.

MSC: 15A15, 11C20, 65F40.

\section{Introduction and main definitions}

$\mathbf{L}$

et $A$ be a $n \times n$ matrix:

$$
\left[A_{n \times n}\right]=\left[\begin{array}{cccc}
a_{11} & a_{12} & \cdots & a_{1 n} \\
a_{21} & a_{22} & \cdots & a_{2 n} \\
\vdots & \vdots & \ddots & \vdots \\
a_{n 1} & a_{n 2} & \cdots & a_{n n}
\end{array}\right]
$$

Definition 1. The determinant of the matrix of order $n \times n$ is the sum

$$
\left|A_{n \times n}\right|=\left|\begin{array}{cccc}
a_{11} & a_{12} & \cdots & a_{1 n} \\
a_{21} & a_{22} & \cdots & a_{2 n} \\
\vdots & \vdots & \ddots & \vdots \\
a_{n 1} & a_{n 2} & \cdots & a_{n n}
\end{array}\right|=\sum_{S_{n}} \varepsilon_{j_{1} j_{2} \ldots j_{n}} \cdot a_{j_{1}} \cdot a_{j_{2}} \cdot \ldots \cdot a_{j_{n}}
$$

ranging over the symmetric permutation group $S_{n}$, where

$$
\varepsilon_{j_{1} j_{2} \ldots j_{n}}=\left\{\begin{array}{c}
+1, \text { if } j_{1} j_{2} \ldots j_{n}, \text { is an even permutation } \\
-1, \text { if } j_{1} j_{2} \ldots j_{n}, \text { is an odd permutation. }
\end{array}\right.
$$

Definition 2. [1] Let $A_{2}=\left[\begin{array}{ll}a_{11} & a_{22} \\ a_{21} & a_{22}\end{array}\right]_{2 \times 2}$ and $B_{2}=\left[\begin{array}{ll}b_{11} & b_{22} \\ b_{21} & b_{22}\end{array}\right]_{2 \times 2}$ are two real matrices of order $2 \times 2$. If $\left|B_{2}\right| \neq 0$ and $b_{i j} \neq 0,(\forall i, j=1,2)$, then the duplex fraction or duplex division of the determinant of $\left|A_{2}\right|$ on $\left|B_{2}\right|$ is defined as follows

$$
\frac{\left|A_{2}\right|}{\overline{\left|B_{2}\right|}}=\frac{\left|\begin{array}{ll}
a_{11} & a_{12} \\
a_{21} & a_{22}
\end{array}\right|}{\left|\begin{array}{ll}
b_{11} & b_{12} \\
b_{21} & b_{22}
\end{array}\right|}=\frac{\left|\begin{array}{ll}
\frac{a_{11}}{b_{11}} & \frac{a_{12}}{b_{12}} \\
\frac{a_{21}}{b_{21}} & \frac{a_{22}}{b_{22}}
\end{array}\right|}{\left|\begin{array}{ll}
b_{11} & b_{12} \\
b_{21} & b_{22}
\end{array}\right|}
$$


Definition 3. [2] Let $A_{3}=\left[\begin{array}{lll}a_{11} & a_{12} & a_{13} \\ a_{21} & a_{22} & a_{23} \\ a_{31} & a_{32} & a_{33}\end{array}\right]_{3 \times 3}$ and $B_{3}=\left[\begin{array}{lll}b_{11} & b_{12} & b_{13} \\ b_{21} & b_{22} & b_{23} \\ b_{31} & b_{32} & b_{33}\end{array}\right]_{3 \times 3}$ are two matrices of order $3 \times 3$ such that $b_{i j} \neq 0,(\forall i, j=1,2,3)$ and $B_{3}$ is doubly nonsingular, then the star fraction of $A_{3}$ on $B_{3}$ is defined as

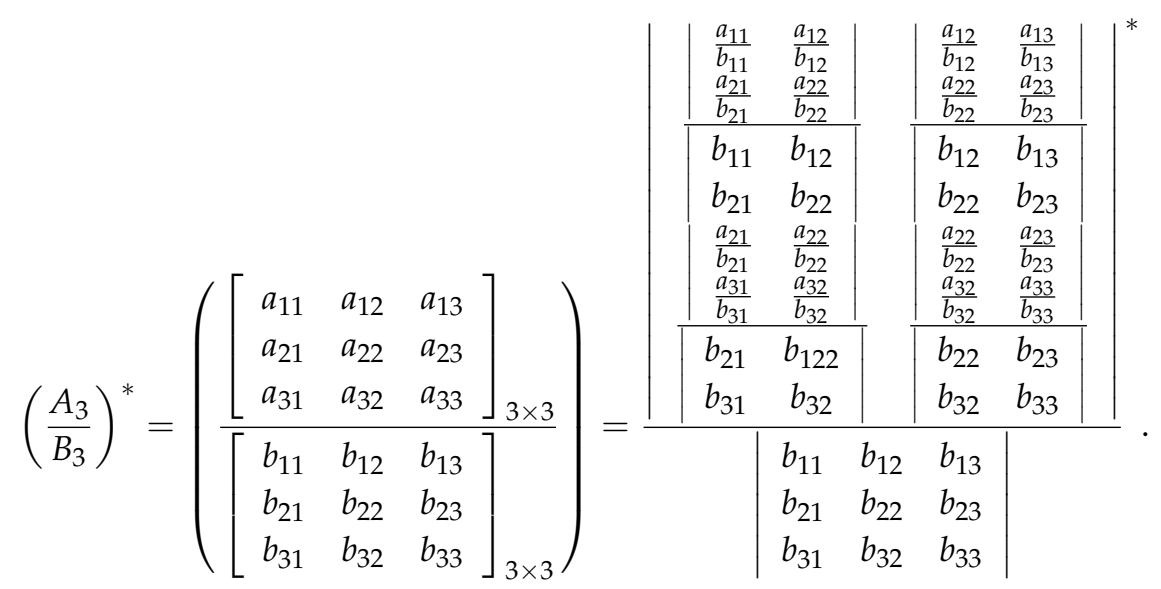

Definition 4. [3] Let $B_{n}=\left[b_{i j}\right]_{n \times n}$ be a square real matrix of order $n$, then the Dodgson's condensation of matrix $B_{n}$ is a $(n-1) \times(n-1)$ matrix defined as:

$$
D C\left(B_{n}\right)=\left[\begin{array}{cc|cc}
\left|\begin{array}{cc}
b_{11} & b_{12} \\
b_{21} & b_{22}
\end{array}\right| & \cdots & \left|\begin{array}{cc}
b_{1(n-1)} & b_{1 n} \\
b_{2(n-1)} & b_{2 n}
\end{array}\right| \\
\vdots & \ddots & \vdots \\
\left|\begin{array}{cc}
b_{(n-1) 1} & b_{(n-1) 2} \\
b_{n 1} & b_{n 2}
\end{array}\right| & \cdots & \left|\begin{array}{cc}
b_{(n-1)(n-1)} & b_{(n-1) n} \\
b_{n(n-1)} & b_{n n}
\end{array}\right|
\end{array}\right]_{(n-1) \times(n-1)}
$$

Definition 5. Let $A_{4}=\left[\begin{array}{llll}a_{11} & a_{12} & a_{13} & a_{14} \\ a_{21} & a_{22} & a_{23} & a_{24} \\ a_{31} & a_{32} & a_{33} & a_{34} \\ a_{41} & a_{42} & a_{43} & a_{44}\end{array}\right]_{4 \times 4}$ be a square matrix of order 4, then the twice Dodgsons's condensation is defined as

$$
\begin{aligned}
& \left.D C\left(A_{4}\right)=\mid \begin{array}{ll}
\left.\left|\begin{array}{ll}
a_{11} & a_{12} \\
a_{21} & a_{22}
\end{array}\right|\left|\begin{array}{ll}
a_{12} & a_{13} \\
a_{22} & a_{23}
\end{array}\right|\left|\begin{array}{ll}
a_{13} & a_{14} \\
a_{23} & a_{24}
\end{array}\right|\right] \\
\left|\begin{array}{ll}
a_{21} & a_{22} \\
a_{31} & a_{32}
\end{array}\right|\left|\begin{array}{ll}
a_{22} & a_{23} \\
a_{32} & a_{33}
\end{array}\right|\left|\begin{array}{ll}
a_{23} & a_{24} \\
a_{33} & a_{34}
\end{array}\right| & , \\
\text { and } D C\left(D C\left(A_{4}\right)\right)= & {\left[\left|\begin{array}{ll}
a_{31} & a_{32} \\
a_{41} & a_{42}
\end{array}\right|\left|\begin{array}{ll}
a_{32} & a_{33} \\
a_{42} & a_{43}
\end{array}\right|\left|\begin{array}{ll}
a_{33} & a_{34} \\
a_{43} & a_{44}
\end{array}\right|\right]_{3 \times 3}} \\
\left|\begin{array}{ll}
a_{21} & a_{22} \\
a_{31} & a_{32}
\end{array}\right|\left|\begin{array}{ll}
a_{11} & a_{12} \\
a_{21} & a_{22}
\end{array}\right|\left|\begin{array}{ll}
a_{22} & a_{23} \\
a_{32} & a_{33}
\end{array}\right|\left|\begin{array}{ll}
a_{22} & a_{13} \\
a_{23}
\end{array}\right||| \begin{array}{ll}
a_{12} & a_{13} \\
a_{22} & a_{23}
\end{array}|| \begin{array}{ll}
a_{13} & a_{14} \\
a_{23} & a_{24}
\end{array}|| \begin{array}{ll}
a_{22} & a_{23} \\
a_{32} & a_{33}
\end{array}|| \begin{array}{ll}
a_{23} & a_{24} \\
a_{33} & a_{34}
\end{array}|| \\
\left|\begin{array}{ll}
a_{21} & a_{22} \\
a_{31} & a_{32}
\end{array}\right|\left|\begin{array}{ll}
a_{22} & a_{23} \\
a_{32} & a_{33}
\end{array}\right||| \begin{array}{ll}
a_{22} & a_{23} \\
a_{32} & a_{33}
\end{array}|| \begin{array}{ll}
a_{23} & a_{24} \\
a_{33} & a_{34}
\end{array}|| \\
\left|\begin{array}{ll}
a_{31} & a_{32} \\
a_{41} & a_{42}
\end{array}\right|\left|\begin{array}{ll}
a_{32} & a_{33} \\
a_{42} & a_{43}
\end{array}\right||| \begin{array}{ll}
a_{32} & a_{33} \\
a_{42} & a_{43}
\end{array}|| \begin{array}{ll}
a_{33} & a_{34} \\
a_{43} & a_{44}
\end{array}||
\end{array}\right]
\end{aligned}
$$


The twice Dodgson's condensation Duplex fraction for the square matrix of order 4 is defined as follows:

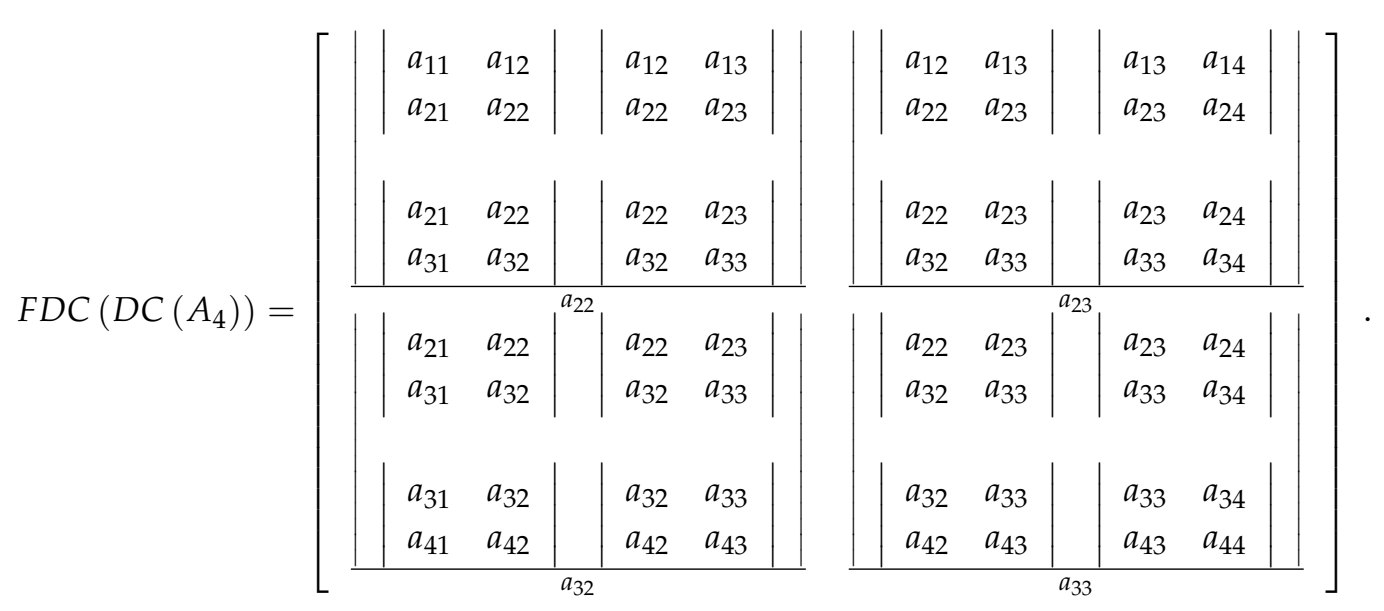

Definition 6. Let $A_{5}=\left[\begin{array}{lllll}a_{11} & a_{12} & a_{13} & a_{14} & a_{15} \\ a_{21} & a_{22} & a_{23} & a_{24} & a_{25} \\ a_{31} & a_{32} & a_{33} & a_{34} & a_{35} \\ a_{41} & a_{42} & a_{43} & a_{44} & a_{45} \\ a_{51} & a_{52} & a_{53} & a_{54} & a_{55}\end{array}\right]_{5 \times 5}$ be a square matrix of order 5 , then the thrice Dodgsons's condensation is defined as follows:

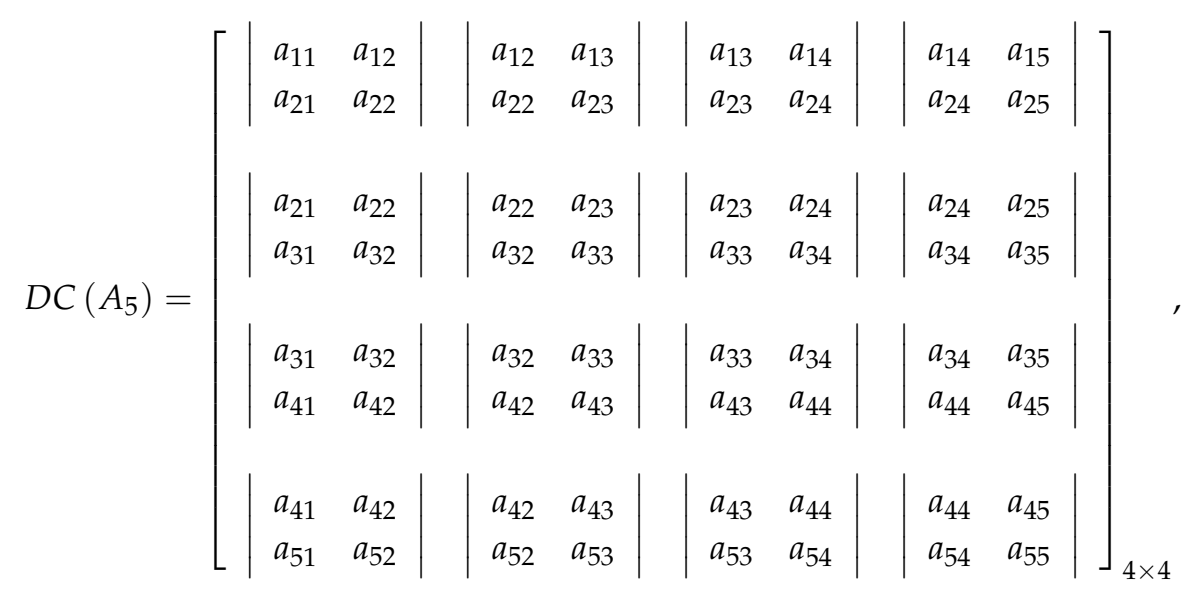

and, $F D C\left(D C\left(A_{5}\right)\right)=$
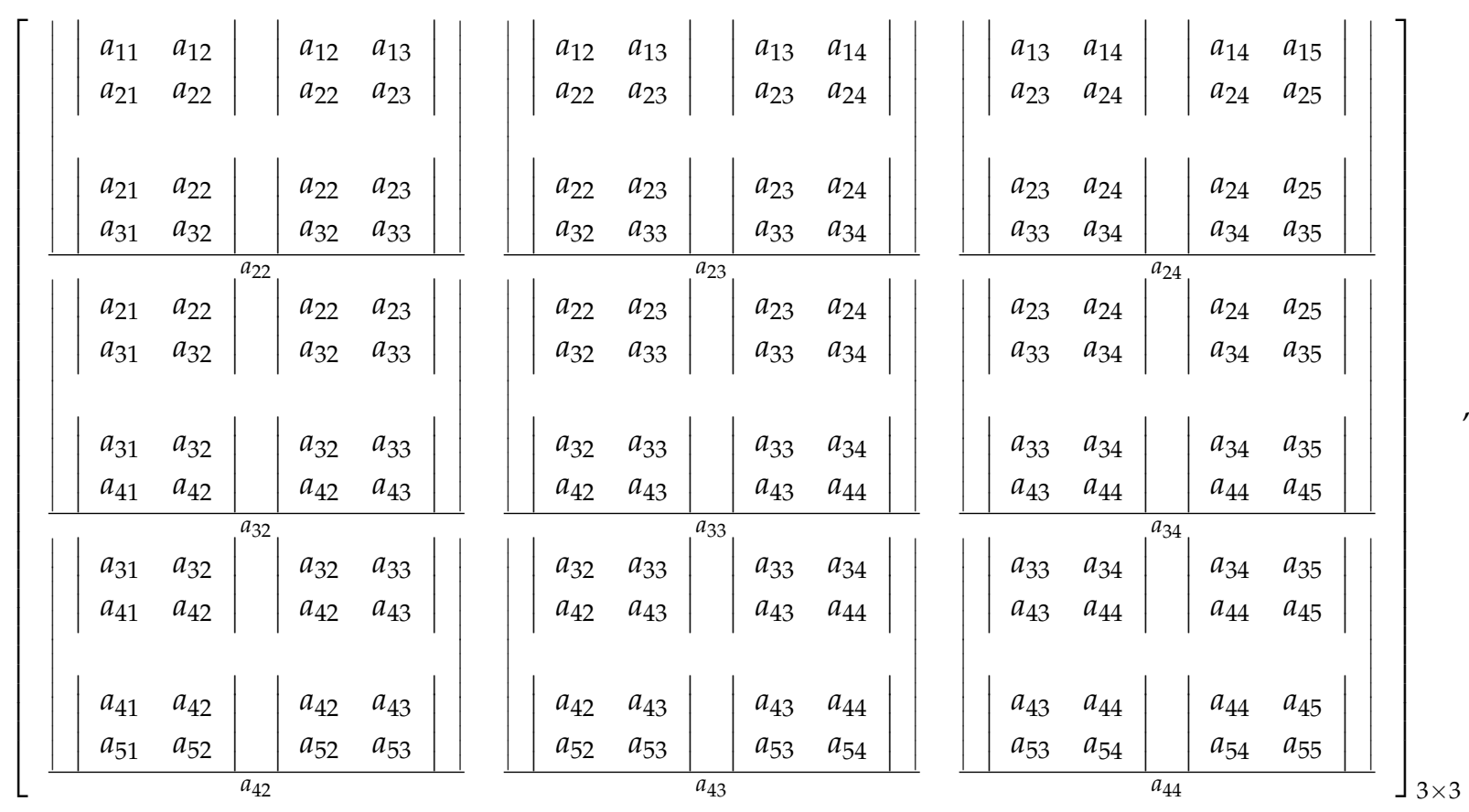
The thrice Dodgson's condensation Duplex fraction for the square matrix of order 5 is defined as follows: $F D C\left(D C\left(D C\left(A_{5}\right)\right)\right)=$
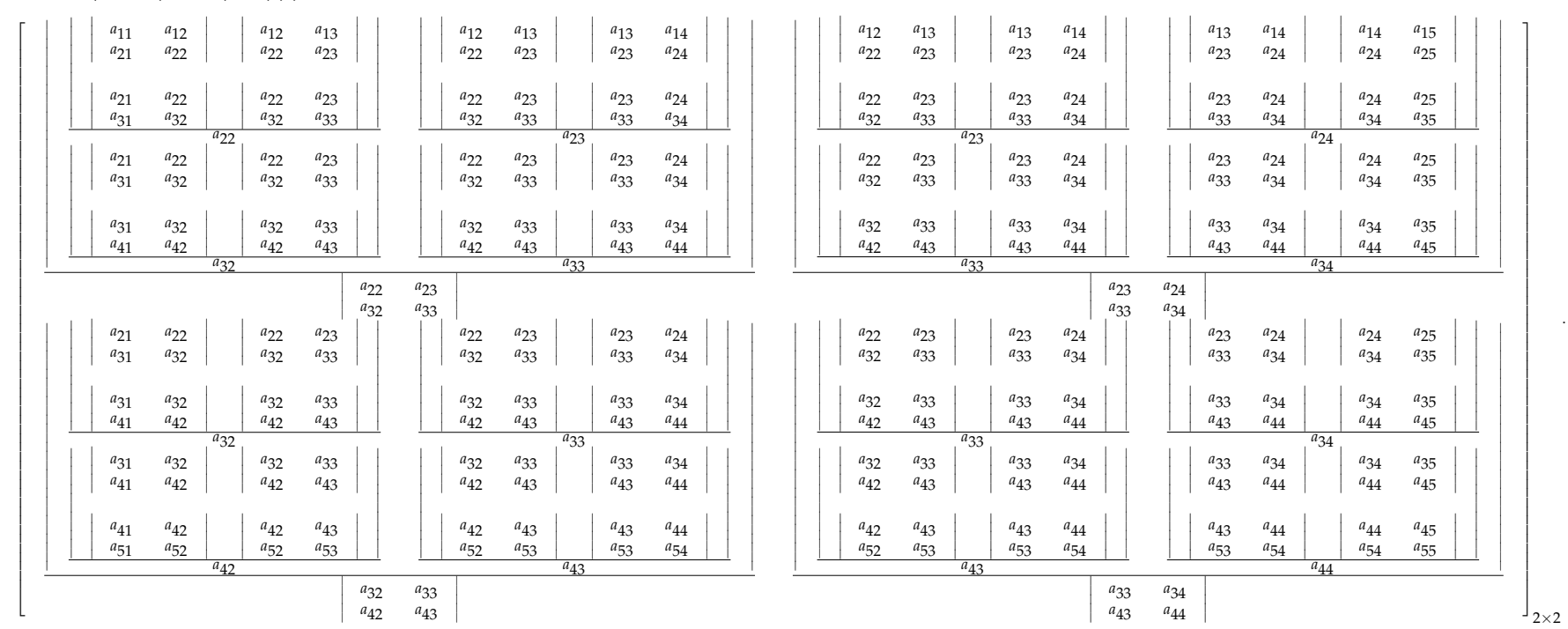

\section{Some useful Lemmas}

To prove our main results we need the following lemmas.

Lemma 7. (Salihu's method [4]) The determinant of the square matrix $A_{n}=\left[\begin{array}{cccc}a_{11} & a_{12} & \cdots & a_{1 n} \\ a_{21} & a_{22} & \cdots & a_{2 n} \\ \vdots & \vdots & \ddots & \vdots \\ a_{n 1} & a_{n 2} & \cdots & a_{n n}\end{array}\right]_{n \times n}$ is equal to:

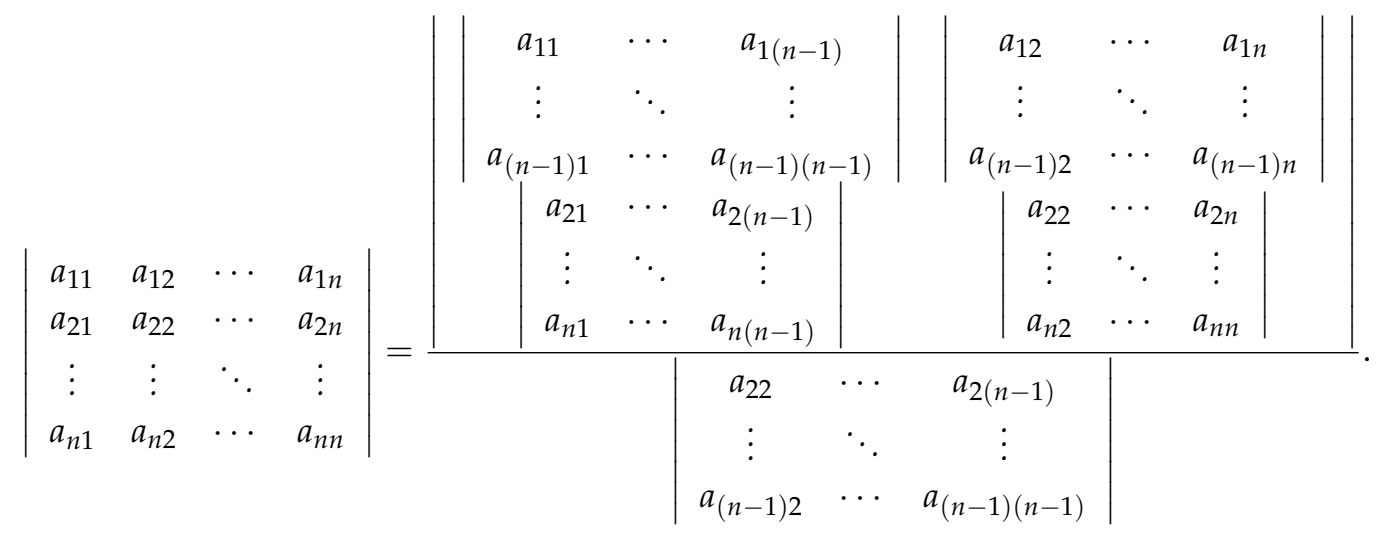

Lemma 8. [1] Given a square matrix

$$
A_{4}=\left[\begin{array}{llll}
a_{11} & a_{12} & a_{13} & a_{14} \\
a_{21} & a_{22} & a_{23} & a_{23} \\
a_{31} & a_{32} & a_{33} & a_{34} \\
a_{41} & a_{42} & a_{43} & a_{44}
\end{array}\right]_{4 \times 4}
$$

of order 4 such that

$$
\left[\begin{array}{ll}
a_{22} & a_{23} \\
a_{32} & a_{33}
\end{array}\right]>0 \text { and }\left|\begin{array}{ll}
a_{22} & a_{23} \\
a_{32} & a_{33}
\end{array}\right| \neq 0
$$

Then

$$
\operatorname{det}\left(A_{4}\right)=\frac{\left|D C\left(D C\left(A_{4}\right)\right)\right|}{\left|\begin{array}{ll}
a_{22} & a_{23} \\
a_{32} & a_{33}
\end{array}\right|}
$$




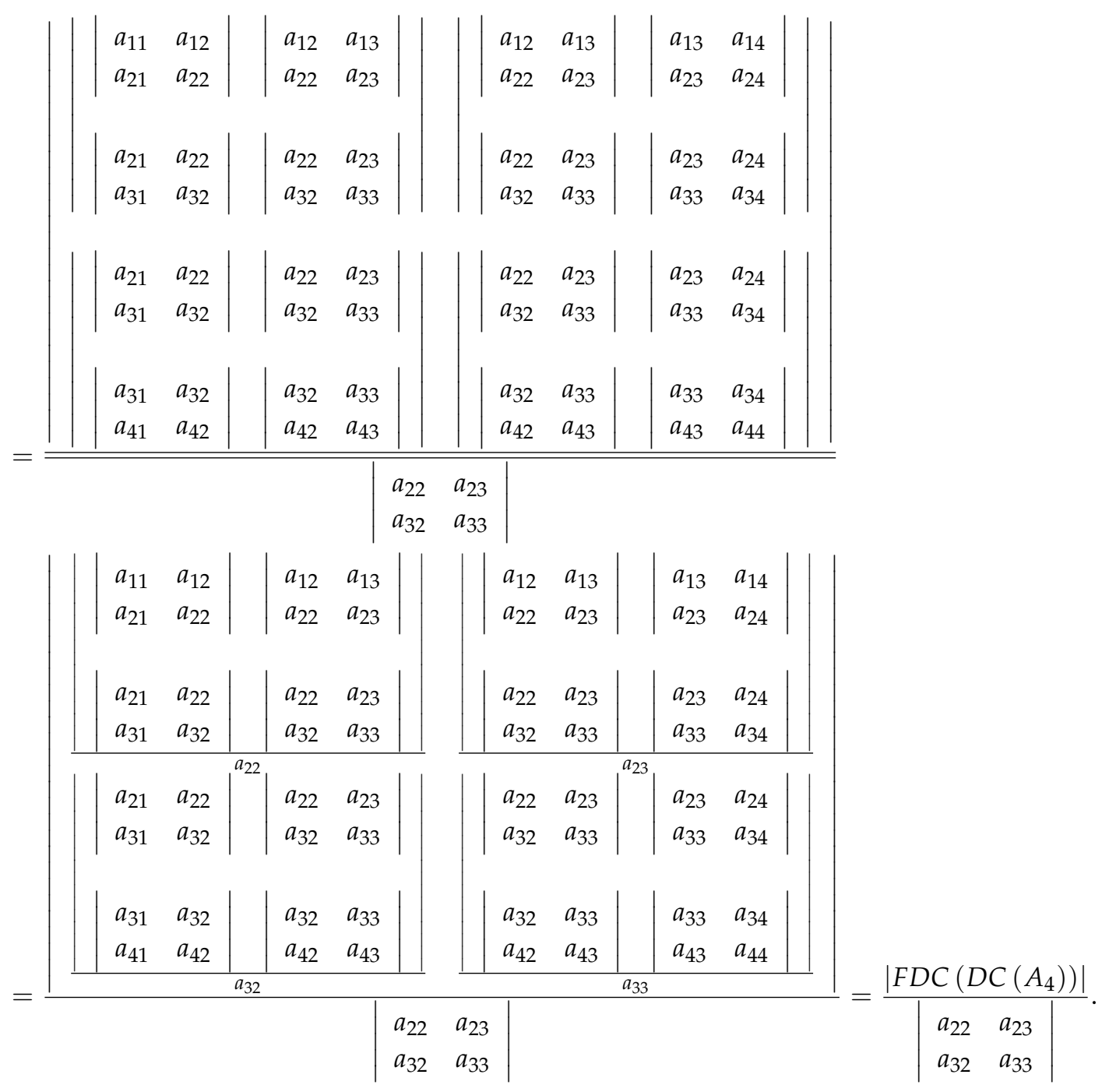

Lemma 9. [2] Consider a square matrix

$$
A_{5}=\left[\begin{array}{lllll}
a_{11} & a_{12} & a_{13} & a_{14} & a_{15} \\
a_{21} & a_{22} & a_{23} & a_{24} & a_{25} \\
a_{31} & a_{32} & a_{33} & a_{34} & a_{35} \\
a_{41} & a_{42} & a_{43} & a_{44} & a_{45} \\
a_{51} & a_{52} & a_{53} & a_{54} & a_{55}
\end{array}\right]_{5 \times 5},
$$

of order 5 , where $\left[\begin{array}{lll}a_{22} & a_{23} & a_{24} \\ a_{32} & a_{33} & a_{34} \\ a_{42} & a_{43} & a_{44}\end{array}\right]$ is a doubly nonsingular matrix with all nonzero elements. Then

$$
\left|A_{5}\right|=\left(\frac{D C\left(D C\left(A_{5}\right)\right)}{\left[\begin{array}{lll}
a_{22} & a_{23} & a_{24} \\
a_{32} & a_{33} & a_{34} \\
a_{42} & a_{43} & a_{44}
\end{array}\right]}\right)^{*}=\frac{\left|D C\left(D C\left(D C\left(A_{5}\right)\right)\right)_{1}\right|}{\left|\begin{array}{lll}
a_{22} & a_{23} & a_{24} \\
a_{32} & a_{33} & a_{34} \\
a_{42} & a_{43} & a_{44} .
\end{array}\right|}
$$

Lemma 10. [5] Suppose that $A$ is a square matrix. Let $B$ be the square matrix obtained from $A$ by interchanging the location of two rows, or interchanging the location of two columns. Then $|A|=-|B|$. 
Lemma 11. [5] Suppose that $A$ is a square matrix. Let $B$ be the square matrix obtained from $A$ by multiplying a single row by the scalar $\alpha$, or by multiplying a single column by the scalar $\alpha$. Then $|A|=\alpha|B|$.

\section{Main Results}

Theorem 12. Given a square matrix $A_{5}=\left[\begin{array}{lllll}a_{11} & a_{12} & a_{13} & a_{14} & a_{15} \\ a_{21} & a_{22} & a_{23} & a_{24} & a_{25} \\ a_{31} & a_{32} & a_{33} & a_{34} & a_{35} \\ a_{41} & a_{42} & a_{43} & a_{44} & a_{45} \\ a_{51} & a_{52} & a_{53} & a_{54} & a_{55}\end{array}\right]_{5 \times 5}$ of order 5 , such that $\left[\begin{array}{lll}a_{22} & a_{23} & a_{24} \\ a_{32} & a_{33} & a_{34} \\ a_{42} & a_{43} & a_{44}\end{array}\right]>0,\left|\begin{array}{lll}a_{22} & a_{23} & a_{24} \\ a_{32} & a_{33} & a_{34} \\ a_{42} & a_{43} & a_{44}\end{array}\right| \neq 0$ and $\left|\begin{array}{ll}a_{22} & a_{23} \\ a_{32} & a_{33}\end{array}\right| \neq 0$, then

$$
\operatorname{det}\left(A_{5}\right)=\frac{\mid \begin{array}{ll}
\left|\left[\operatorname{FDC}\left(D C\left(A_{4}\right)\right)_{1}\right]^{*}\right| & \left|\left[\operatorname{FDC}\left(D C\left(A_{4}\right)\right)_{2}\right]^{*}\right| \\
\left|\left[\operatorname{FDC}\left(D C\left(A_{4}\right)\right)_{3}\right]^{*}\right| & \left|\left[\operatorname{FDC}\left(D C\left(A_{4}\right)\right)_{4}\right]^{*}\right|
\end{array}}{\left|\begin{array}{lll}
a_{22} & a_{23} & a_{24} \\
a_{32} & a_{33} & a_{34} \\
a_{42} & a_{43} & a_{44}
\end{array}\right| \cdot\left|\begin{array}{ll}
a_{22} & a_{23} \\
a_{32} & a_{33}
\end{array}\right|^{2}}
$$

Proof. By Lemma 7, we have:

$$
\left|A_{5}\right|=\left|\begin{array}{lllll}
a_{11} & a_{12} & a_{13} & a_{14} & a_{15} \\
a_{21} & a_{22} & a_{23} & a_{24} & a_{25} \\
a_{31} & a_{32} & a_{33} & a_{34} & a_{35} \\
a_{41} & a_{42} & a_{43} & a_{44} & a_{45} \\
a_{51} & a_{52} & a_{53} & a_{54} & a_{55}
\end{array}\right|=\frac{\left|\begin{array}{llll}
a_{11} & a_{12} & a_{13} & a_{14} \\
a_{21} & a_{22} & a_{23} & a_{24} \\
a_{31} & a_{32} & a_{33} & a_{34} \\
a_{41} & a_{42} & a_{43} & a_{44}
\end{array}\right|\left|\begin{array}{llll}
a_{12} & a_{13} & a_{14} & a_{15} \\
a_{22} & a_{23} & a_{24} & a_{25} \\
a_{32} & a_{33} & a_{34} & a_{35} \\
a_{42} & a_{43} & a_{44} & a_{45}
\end{array}\right|}{\left|\begin{array}{llll}
a_{21} & a_{22} & a_{23} & a_{24} \\
a_{31} & a_{32} & a_{33} & a_{34} \\
a_{41} & a_{42} & a_{43} & a_{44} \\
a_{51} & a_{52} & a_{53} & a_{54}
\end{array}\right|\left|\begin{array}{llll}
a_{22} & a_{23} & a_{24} & a_{25} \\
a_{32} & a_{33} & a_{34} & a_{35} \\
a_{42} & a_{43} & a_{44} & a_{45} \\
a_{52} & a_{53} & a_{54} & a_{55}
\end{array}\right|},
$$

by Lemma 10, we have

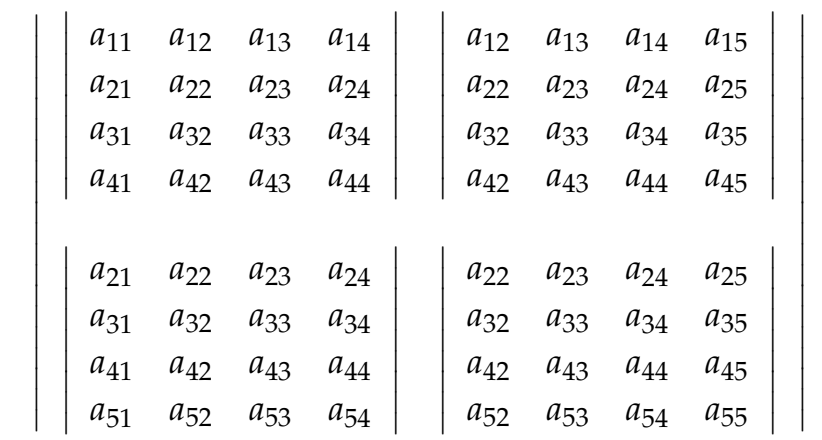

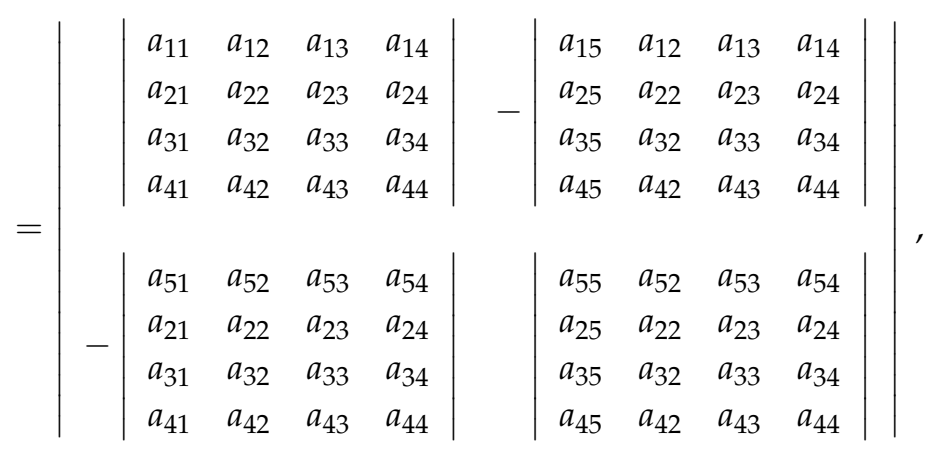


by Lemma 8 , we have:

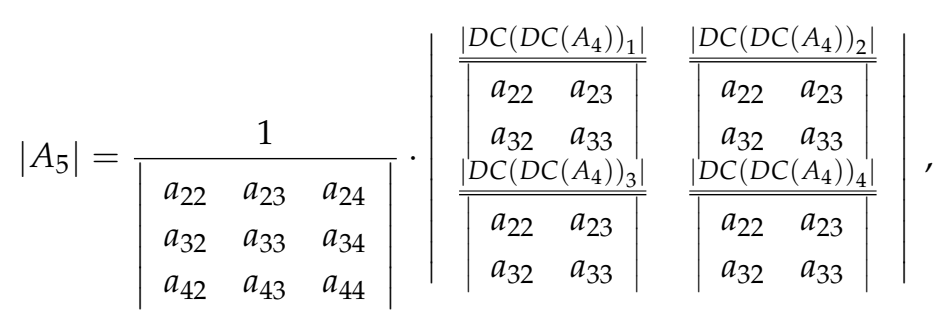

using Definition 6 and Lemma 11, we obtain

$$
\begin{aligned}
& \left|A_{5}\right|=\frac{1}{\left|\begin{array}{lll}
a_{22} & a_{23} & a_{24} \\
a_{32} & a_{33} & a_{34} \\
a_{42} & a_{43} & a_{44}
\end{array}\right|} \cdot \frac{1}{\left|\begin{array}{ll}
a_{22} & a_{23} \\
a_{32} & a_{33}
\end{array}\right|^{2}} \cdot \mid \begin{array}{ll}
\left|\left[\operatorname{FDC}\left(D C\left(A_{4}\right)\right)_{1}\right]^{*}\right| & \left|\left[\operatorname{FDC}\left(D C\left(A_{4}\right)\right)_{2}\right]^{*}\right| \\
\left|\left[\operatorname{FDC}\left(D C\left(A_{4}\right)\right)_{3}\right]^{*}\right| & \left|\left[\operatorname{FDC}\left(D C\left(A_{4}\right)\right)_{4}\right]^{*}\right|
\end{array} \\
& =\frac{\mid \begin{array}{ll}
\left|\left[F D C\left(D C\left(A_{4}\right)\right)_{1}\right]^{*}\right| & \left|\left[F D C\left(D C\left(A_{4}\right)\right)_{2}\right]^{*}\right| \\
\left|\left[F D C\left(D C\left(A_{4}\right)\right)_{3}\right]^{*}\right| & \left|\left[F D C\left(D C\left(A_{4}\right)\right)_{4}\right]^{*}\right|
\end{array}}{\left|\begin{array}{lll}
a_{22} & a_{23} & a_{24} \\
a_{32} & a_{33} & a_{34} \\
a_{42} & a_{43} & a_{44}
\end{array}\right| \cdot\left|\begin{array}{ll}
a_{22} & a_{23} \\
a_{32} & a_{33}
\end{array}\right|^{2}}
\end{aligned}
$$

This complete the prove.

Example 1. Let $A=\left[\begin{array}{lllll}2 & 1 & 4 & 1 & 3 \\ 1 & 4 & 3 & 2 & 1 \\ 2 & 5 & 2 & 3 & 2 \\ 1 & 1 & 4 & 1 & 2 \\ 2 & 3 & 1 & 4 & 1\end{array}\right]$ be a square matric of order 5, then by using Theorem 12, we have

$$
\begin{aligned}
& \left|A_{5}\right|=\left|\begin{array}{lllll}
2 & 1 & 4 & 1 & 3 \\
1 & 4 & 3 & 2 & 1 \\
2 & 5 & 2 & 3 & 2 \\
1 & 1 & 4 & 1 & 2 \\
2 & 3 & 1 & 4 & 1
\end{array}\right|
\end{aligned}
$$

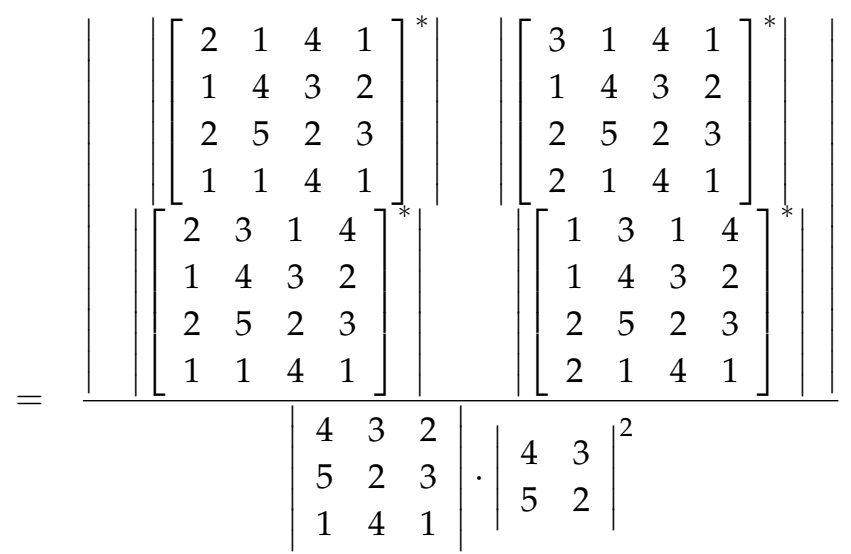

$$
\begin{aligned}
& =\frac{\left|\begin{array}{rr}
70 & 70 \\
-175 & -350
\end{array}\right|}{-10 \cdot(-7)^{2}} \\
& =\frac{-12250}{-490}=25 \text {. }
\end{aligned}
$$


Theorem 13. Given a square matrix

$$
A_{6}=\left[\begin{array}{llllll}
a_{11} & a_{12} & a_{13} & a_{14} & a_{15} & a_{16} \\
a_{21} & a_{22} & a_{23} & a_{24} & a_{25} & a_{26} \\
a_{31} & a_{32} & a_{33} & a_{34} & a_{35} & a_{36} \\
a_{41} & a_{42} & a_{43} & a_{44} & a_{45} & a_{46} \\
a_{51} & a_{52} & a_{53} & a_{54} & a_{55} & a_{56} \\
a_{61} & a_{62} & a_{63} & a_{64} & a_{65} & a_{66}
\end{array}\right]_{6 \times 6}
$$

of order 6 such that

$$
\left[\begin{array}{llll}
a_{22} & a_{23} & a_{24} & a_{25} \\
a_{32} & a_{33} & a_{34} & a_{35} \\
a_{42} & a_{43} & a_{44} & a_{45} \\
a_{52} & a_{53} & a_{54} & a_{55}
\end{array}\right]>0,\left|\begin{array}{llll}
a_{22} & a_{23} & a_{24} & a_{25} \\
a_{32} & a_{33} & a_{34} & a_{35} \\
a_{42} & a_{43} & a_{44} & a_{45} \\
a_{52} & a_{53} & a_{54} & a_{55}
\end{array}\right| \neq 0 \text { and }\left|\begin{array}{lll}
a_{33} & a_{34} & a_{35} \\
a_{43} & a_{44} & a_{45} \\
a_{53} & a_{54} & a_{55}
\end{array}\right| \neq 0,
$$

then

$$
\operatorname{det}\left(A_{6}\right)=\frac{\mid \begin{array}{lll}
\left|\left[F D C\left(D C\left(D C\left(A_{5}\right)\right)\right)_{1}\right]^{*}\right| & \mid\left[F D C\left(D C\left(D C\left(A_{5}\right)\right)_{2}\right]^{*} \mid\right. \\
\left|\left[F D C\left(D C\left(D C\left(A_{5}\right)\right)\right)_{3}\right]^{*}\right| & \mid\left[F D C\left(D C\left(D C\left(A_{5}\right)\right)_{4}\right]^{*} \mid\right.
\end{array}}{\left|\begin{array}{llll}
a_{22} & a_{23} & a_{24} & a_{25} \\
a_{32} & a_{33} & a_{34} & a_{35} \\
a_{42} & a_{43} & a_{44} & a_{45} \\
a_{52} & a_{53} & a_{54} & a_{55}
\end{array}\right| \cdot\left|\begin{array}{lll}
a_{22} & a_{23} & a_{24} \\
a_{32} & a_{33} & a_{34} \\
a_{42} & a_{43} & a_{44}
\end{array}\right|^{2}}
$$

Proof. By Lemma 7, we have

$$
\begin{aligned}
\left|A_{6}\right|=\left|\begin{array}{llllll}
a_{11} & a_{12} & a_{13} & a_{14} & a_{15} & a_{16} \\
a_{21} & a_{22} & a_{23} & a_{24} & a_{25} & a_{26} \\
a_{31} & a_{32} & a_{33} & a_{34} & a_{35} & a_{36} \\
a_{41} & a_{42} & a_{43} & a_{44} & a_{45} & a_{46} \\
a_{51} & a_{52} & a_{53} & a_{54} & a_{55} & a_{56} \\
a_{61} & a_{62} & a_{63} & a_{64} & a_{65} & a_{66}
\end{array}\right| \\
\left|\begin{array}{lllll}
a_{11} & a_{12} & a_{13} & a_{14} & a_{15} \\
a_{21} & a_{22} & a_{23} & a_{24} & a_{25} \\
a_{31} & a_{32} & a_{33} & a_{34} & a_{35} \\
a_{41} & a_{42} & a_{43} & a_{44} & a_{45} \\
a_{51} & a_{52} & a_{53} & a_{54} & a_{55}
\end{array}\right|\left|\begin{array}{lllllll}
a_{12} & a_{13} & a_{14} & a_{15} & a_{16} \\
a_{22} & a_{23} & a_{24} & a_{25} & a_{26} \\
a_{32} & a_{33} & a_{34} & a_{35} & a_{36} \\
a_{42} & a_{43} & a_{44} & a_{45} & a_{46} \\
a_{52} & a_{53} & a_{54} & a_{55} & a_{56}
\end{array}\right| \\
\left|\begin{array}{lllllllll}
a_{21} & a_{22} & a_{23} & a_{24} & a_{25} \\
a_{31} & a_{32} & a_{33} & a_{34} & a_{35} \\
a_{41} & a_{42} & a_{43} & a_{44} & a_{45} \\
a_{51} & a_{52} & a_{53} & a_{54} & a_{55} \\
a_{61} & a_{62} & a_{63} & a_{64} & a_{55}
\end{array}\right|\left|\begin{array}{lllllll}
a_{22} & a_{23} & a_{24} & a_{25} & a_{26} \\
a_{32} & a_{33} & a_{34} & a_{35} & a_{36} \\
a_{42} & a_{43} & a_{44} & a_{45} & a_{46} \\
a_{52} & a_{53} & a_{54} & a_{55} & a_{56} \\
a_{62} & a_{63} & a_{64} & a_{65} & a_{56}
\end{array}\right|
\end{aligned}
$$


by Lemma 10, we know that

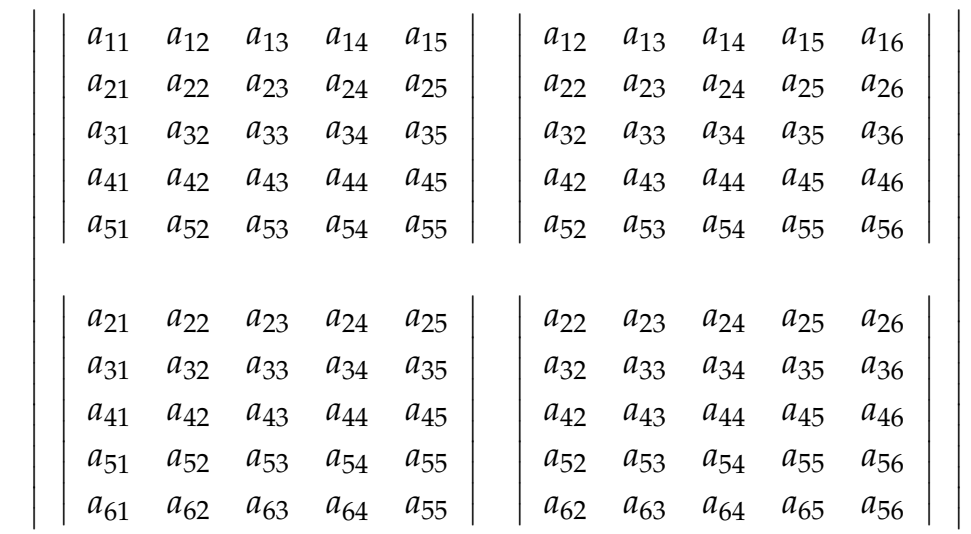

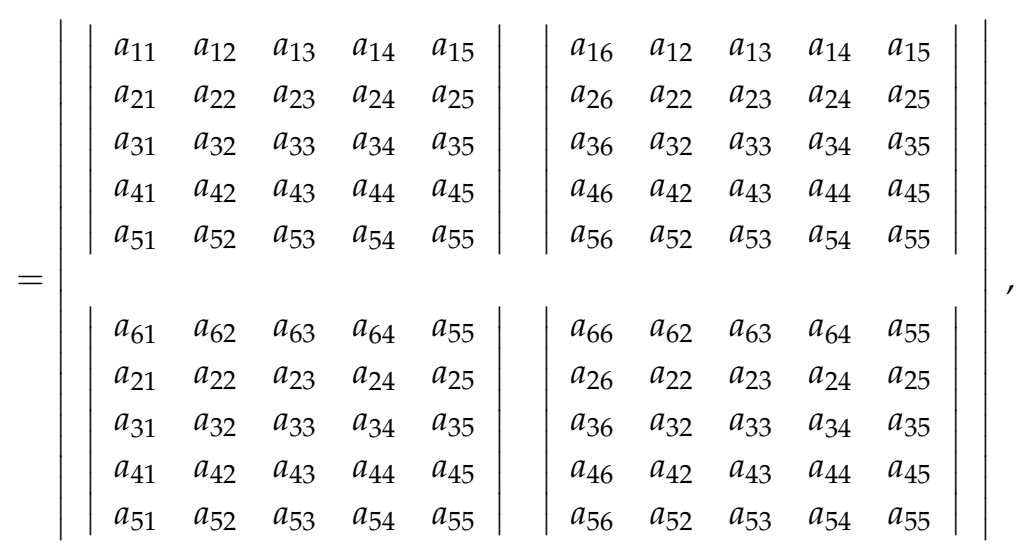

using Lemma 9, we have

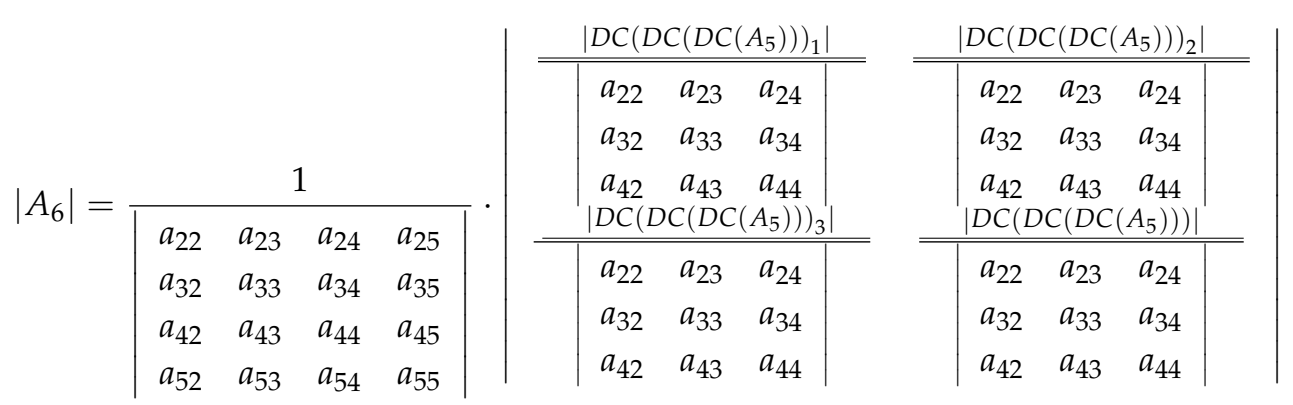

using Definition 6 and Lemma 11, we obtain

$$
\begin{aligned}
& \left|A_{6}\right|=\frac{1}{\left|\begin{array}{llll}
a_{22} & a_{23} & a_{24} & a_{25} \\
a_{32} & a_{33} & a_{34} & a_{35} \\
a_{42} & a_{43} & a_{44} & a_{45} \\
a_{52} & a_{53} & a_{54} & a_{55}
\end{array}\right|} \cdot \frac{1}{\left|\begin{array}{lll}
a_{22} & a_{23} & a_{24} \\
a_{32} & a_{33} & a_{34} \\
a_{42} & a_{43} & a_{44}
\end{array}\right|^{2}} \\
& \cdot\left|\begin{array}{ll}
\left|\left[F D C\left(D C\left(D C\left(A_{5}\right)\right)\right)_{1}\right]^{*}\right| & \mid\left[F D C\left(D C\left(D C\left(A_{5}\right)\right)_{2}\right]^{*} \mid\right. \\
\left|\left[F D C\left(D C\left(D C\left(A_{5}\right)\right)\right)_{3}\right]^{*}\right| & \mid\left[F D C\left(D C\left(D C\left(A_{5}\right)\right)_{4}\right]^{*} \mid\right.
\end{array}\right|
\end{aligned}
$$

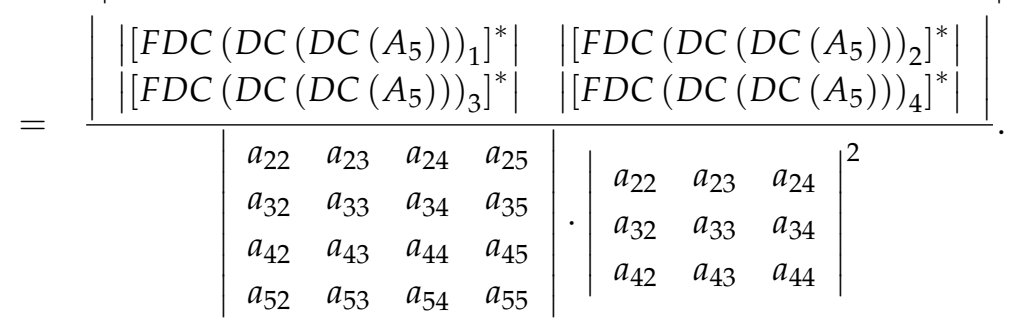

This complete the prove. 
Example 2. Let $A\left[\begin{array}{llllll}1 & 4 & 2 & 1 & 3 & 2 \\ 2 & 1 & 1 & 4 & 2 & 3 \\ 2 & 3 & 4 & 1 & 1 & 2 \\ 3 & 1 & 4 & 2 & 1 & 4 \\ 1 & 4 & 2 & 3 & 4 & 3 \\ 4 & 1 & 1 & 2 & 1 & 3\end{array}\right]$ be a square matrix of order 6 , then by using Theorem 13, we have

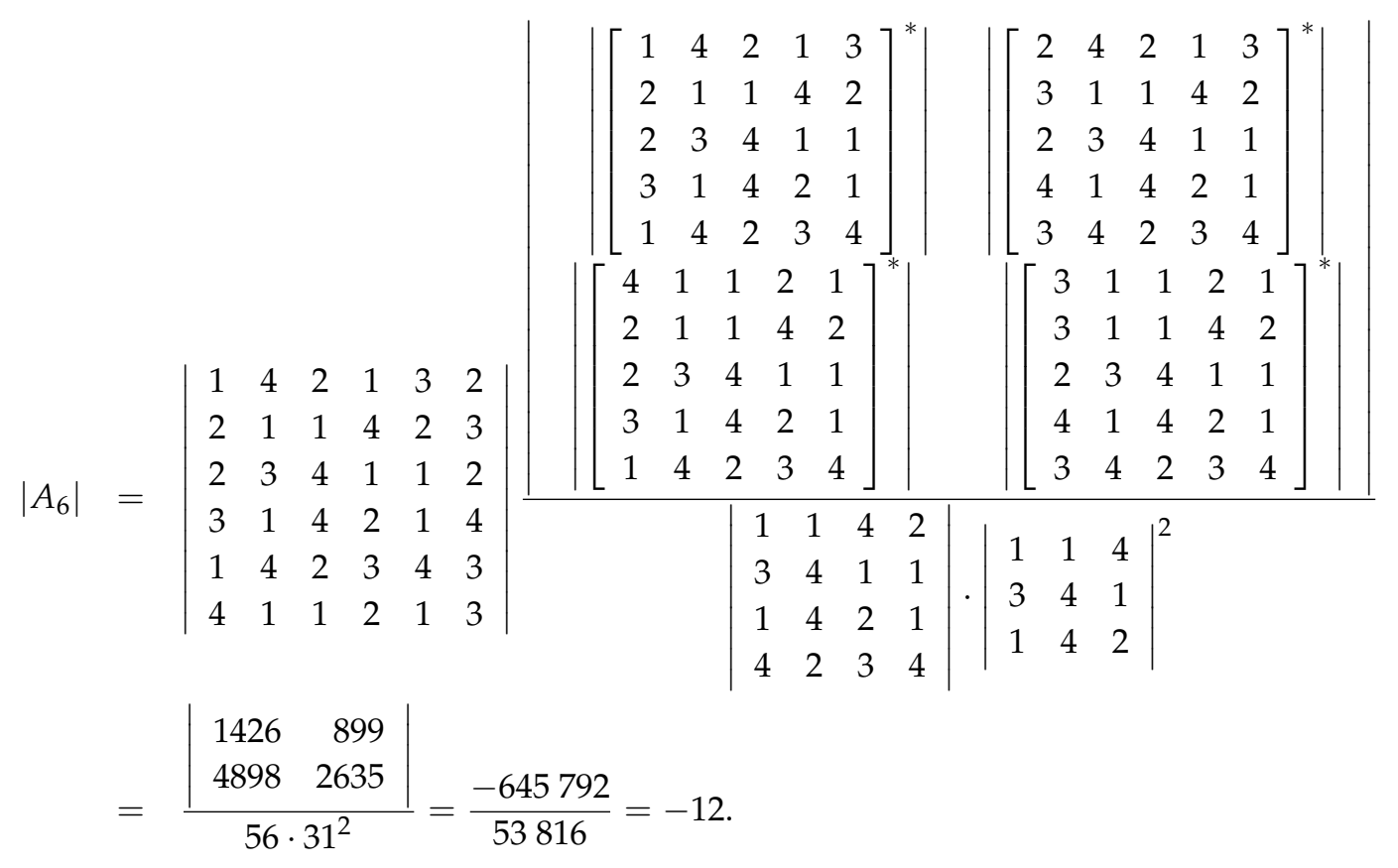

Conflicts of Interest: “The author declare no conflict of interest."

\section{References}

[1] Farhadian, R. (2017). On A Method to Compute the Determinant of Square Matrices of Order Four. International Journal of Scientific and Innovative Mathematical Research 5(4)5.

[2] Farhadian, R. (2018). A Method to Compute the Determinant of $5 \times 5$ Matrix. Open Journal of Mathematical Sciences 2(1), 156-163.

[3] Dogdson, C. L. (1866). Condensation of determinants, being a new and brief method for computing their numerical values. Proc. Royal. Soc. London, 15(150-155), 342.

[4] Salihu. A. (2012). Method to Calculate Determinants of $n \times n(n \geq 3)$ Matrix, by Reducing Determinants to 2nd Order. International Journal of Algebra 6(19),913-917.

[5] Strang, G. (1993). Introduction to linear algebra (Vol. 3). Wellesley, MA: Wellesley-Cambridge Press. 